area review committee, to include a family doctor, probation officer, and representatives of other relevant bodies.

The report emphasizes the key role of the consultant in accident and emergency departments in making the diagnosis, collecting data, and ensuring that attendances at different hospitals and different parts of one hospital are recorded, so that the suspicion of child abuse can be aroused. For a battering family may go from hospital to hospital, or from one department of a hospital to another, turning up at one time with the child burnt, another time with a fracture, and another time with failure to thrive. As the report points out, research is needed into the effect of different types of management, including criminal proceedings. Methods of prevention also need study. They might include better family planning, the protection of siblings (who are at considerable risk), and instruction in schools about the development and needs of children.

The recommendations are eminently sensible. Increases in staff will be needed to carry out some of them. But children's lives are at stake.

1 Caffey, J., American Fournal of Diseases of Children, 1972, 124, 161.

2 Tunbridge Wells Study Group, Non-accidental Injury to Children, 1973.

3 Kempe, C. H., Archives of Disease in Childhood, 1971, 46, 28.

4 Solomon, T., Symposium on Child Abuse, Pediatrics, Supplement 51, No. 4 , pt. 2,773 .

5 Adelson, 2, 77.

${ }^{6}$ Medical Aspects of Home Hazards, ed. A. Nelson, London, Medical

Commission on Accident Prevention, 1972.

\section{Siøgren's Syndrome}

"Commoner than hitherto realized" is a hard-worked cliché in medical writing, often enthusiastically applied to descriptions of clinical rarities. But a condition for which it appears to be well justified is Sjøgren's syndrome, first clearly described by Henriks Sjøgren, a Swedish ophthalmologist, in $1933 .{ }^{1}$ It is defined as a triad of dry mouth (xerostomia), dry eyes (xerophthalmia), and a connective tissue disease-usually rheumatoid arthritis.

During the past decade the extent to which the disease affects a variety of organs has become apparent. ${ }^{2-4}$ The characteristic lymphoid infiltration is not confined to the salivary and lachrymal glands but occurs also in the nasal cavity, pharynx, larynx, trachea, bronchi, sweat glands, and kidney, with such diverse effects as recurrent epistaxis, bronchitis, recurrent chest infections, and renal tubular abnormalities as well as the better known ocular and oral symptoms.

A characteristic of the syndrome is the presence of hypergammaglobulinaemia and of a variety of circulating autoantibodies, both organ-specific and non-organ-specific. Its clinical and immunological features have recently been reviewed in the B.M.F. ${ }^{5}$ and in a monograph by M. A. Shearn. ${ }^{6}$ The exact prevalence of the syndrome and in particular the extent of its relationship with rheumatoid arthritis are not precisely known. Most series to date have been highly selected and have concentrated on the more florid forms of the disease.

More work on it has now been reported in two articles by $\mathrm{K}$. Whaley and his colleagues ${ }^{7}$ from Glasgow in a six-year study of 171 patients with Sjøgren's syndrome. Of these, 94 patients had rheumatoid arthritis, 4 had systemic lupus erythematosus, 1 had progressive systemic sclerosis, and 1 had psoriatic arthritis. In 71 patients Sjøgren's syndrome was unassociated with rheumatoid arthritis or other connective tissue or rheumatic diseases (the so-called sicca syndrome).
The mean age was 57 years and $89 \%$ were female-a sex incidence closer to that of systemic lupus erythematosus than rheumatoid arthritis.

Some interesting clinical points emerged. While the majority of patients complained of ocular symptoms, few were diagnosed by their general practitioner or consultant physician as having the disease. The symptoms of keratoconjunctivitis sicca were commoner in patients with the sicca syndrome than in those with rheumatoid arthritis, the commonest complaints being sensations of "burning" or a "foreign body." While the diagnostic value of the Schirmer test for secretion of tears was stressed, diminished tear secretion was also found in hot dry atmospheres and with increasing frequency in the elderly. In elderly patients the keratoconjunctivitis sicca does not appear to reflect subclinical inflammation of the lachrymal glands and is not associated with the presence of circulating autoantibodies. ${ }^{8}$ Artificial tear drops ( $0.5 \%$ carboxymethylcellulose) were satisfactory in controlling ocular symptoms in most of the patients.

The symptoms and signs of xerostomia were more variable and were often intermittent. A history of increased intake of fluid was common. The diagnostic value of biopsy of labial salivary glands was again confirmed. ${ }^{9}$ Lymphocytic inflammation of the salivary gland was found in $62 \%$ of the patients with the sicca syndrome, $70 \%$ of patients with rheumatoid arthritis and Sjøgren's syndrome, and, notably, in 19\% of patients with rheumatoid arthritis alone. Enlargement of the salivary glands was seen in 37 patients. The parotid glands were most frequently affected, and unilateral and intermittent swelling was common. Rates of salivary flow were reduced in all groups of patients with this syndrome, but the flow rate is limited as an index of salivary gland function because the variation is so wide in normal persons.

The study re-emphasized how commonly the upper respiratory tract is affected in this disease. Whaley and his colleagues suggest that postoperative suppurative parotitis might be more prevalent in patients with Sjøgren's syndrome than in general. On similar reasoning it would also seem likely that postoperative atelectasis and chest infection are commoner in this group of patients.

Of 15 patients assessed for renal tubular function one had overt renal tubular acidosis, five had latent renal tubular acidosis, and three had impaired renal concentrating ability. One patient had generalized aminoaciduria.

Immunological studies again confirmed the high incidence of non-organ-specific and of organ-specific antibodies, though it is interesting to note that there was no clear association between organ-specific autoimmune diseases and Sjogren's syndrome.

G. R. V. Hughes and Whaley have advocated the routine testing of patients with rheumatic diseases for evidence of conjunctivitis sicca. ${ }^{5}$ Apart from the considerable disability associated with Sjøgren's syndrome two other features deserve particular attention. Over the past two decades some cases of malignant disease of the reticuloendothelial system have been reported in association with it. ${ }^{10-12}$ The incidence is unknown, though rare, and this complication appears to be more common in patients with the sicca syndrome alone. Of interest was the finding that of the 7 patients in the Glasgow series with disease of the lachrymal glands one developed lymphosarcoma. The only patient in an earlier series ${ }^{3}$ who had enlarged lachrymal glands subsequently developed reticulum cell sarcoma. The second feature to be noted is the tendency of these patients to develop drug allergies. B. O. Williams and colleagues $^{13}$ noted that the incidence of penicillin allergy in patients with Sjøgren's syndrome was $40 \%$ as compared with 
$16 \%$ in those with rheumatoid arthritis alone. The more widespread recognition of this syndrome may distinguish a group of patients in whom extra therapeutic caution is required.

'Sjøgren, H., Acta Ophthalmologica, Suppl. No. 2, p. 1, 1933.

Bloch, K. J., and Bunim, J. J., Fournal of Chronic Diseases, 1963, 16, 915.
Bloch, K. J., Buchanan, W. W., Wohl, M. J., and Bunim, J. J., Medicine, 1965, K. J., Buch

Whaley, K., and Buchanan, W. W., in Modern Trends in Rheumatology, vol. 2, ed. A. G. S. Hill. London, Butterworth, 1971

${ }^{5}$ Hughes, G. R. V., and Whaley, K., British Medical fournal, 1972, 4, 533.

- Shearn, M. A., Sipgren's Syndrome. Philadelphia, Saunders, 1971.

Whaley, K., et al., Quarterly fournal of Medicine, 1973, 42, 279, 513

Whaley, K., et al., Age and Ageing, 1972, 1, 197.

Chisholm, D. M., and Mason, D. K., Fournal of Clinical Pathology, 1968, 21, 656 .

${ }^{0}$ Rothman, S., Block, M., and Hauser, F. V., Archives of Dermatology and Syphilology, 1951, 63, 642.

11 Talal, N., and Bunim, J. J., American fournal of Medicine, 1964, 36, 529.

Talal, N., Sokoloff, L., and Barth, W. F., American fournal of Medicine, $1967,43,50$.

13 Williams, B. O., et al., Annals of Rheumatic Diseases, 1969, 28, 670.

\section{Idiopathic Cardiomyopathy in Infants}

The term "cardiomyopathy" was introduced by Brigden in $1957^{1}$ to describe isolated non-coronary myocardial disease of obscure aetiology. Over the succeeding years the term has been employed to include almost any cardiac disease but restriction is clearly necessary. ${ }^{2}$ It has also been suggested that terms "primary" and "idiopathic" should be dropped, ${ }^{3}$ and that the term "cardiomyopathy" should be reserved for disorders of heart muscle of unknown cause or association."

The cardiomyopathies can be subdivided clinically into two major types: congestive and hypertrophic (with or without obstruction), and two rarer types, constrictive and obliterative. $^{5}$ By definition the aetiology is unknown, and much research has been concentrated on elucidating it. Viral infection has been suggested as a possible cause, particularly of the congestive type, but proof has often been lacking. ${ }^{6} \mathrm{M}$. B. Gardner and colleagues ${ }^{7}$ successfully demonstrated virus-like particles in a patient suffering from cardiomyopathy. Many reported cases of cardiomyopathy have been described in adults, but to gain insight into the disease process and to find a cause much can be learned from focusing attention on newborn babies and infants because of their relatively short history.

Under the title "idiopathic cardiomyopathy" R. Doshi and K. V. Lodge ${ }^{8}$ recently described the cases of nine infants. Their ages varied between 14 days and 9 months; there were four girls and five boys. Two boys were siblings. The common mode of death was circulatory failure (five patients), or sudden collapse (three patients), and in four instances was accompanied or precipitated by bronchopneumonia. At necropsy the hearts showed no abnormality other than dilatation and hypertrophy of all chambers. In three cases mainly the left ventricle was hypertrophied, in two cases mainly the right, and in the remaining four patients equal hypertrophy of right and left ventricle was found.

Histological examination of the myocardium showed irregular swelling and distortion of fibres often widely separated from one another, with occasionally amorphous pink-staining material between them. Some fibres were vacuolated, others necrotic, and still others showed a perinuclear halo which contained periodic-acid-Schiff-positive material. Fibrosis or inflammatory cell infiltrates were absent.

Immunofluorescent tests for bound gammaglobulin showed none in any of the cases. Degenerative cellular changes in the large ganglia were noted, but similar changes were observed in 10 out of the 11 control patients who died from causes other than cardiomyopathy. These changes were interpreted as not being due to virus disease ${ }^{8}$ but possibly due to terminal anoxia. This interpretation was strengthened by the fact that these changes in the ganglia were more recent than the myocardial changes, and also they were absent in a further group of six infants of the control series who did not die in heart failure. Viral cultures of necropsy material were undertaken in six of the nine infants, but all were negative. In three of four mothers there were significent antibody titres in the serum against viruses which included mumps viruses, measles, influenza $\mathrm{A}$, adenovirus, influenza, and rubella. No antiheart or antinuclear antibodies were detected in the mothers' sera.

As the disease appeared so early in life, an intrauterine viral cause was a possibility ${ }^{8}$ It was also postulated that the myocardial damage might be caused by a complex immune mechanism resulting from virus or virus antibodies crossing the placenta. In older infants viral infection may have occurred after birth, the maternal antibodies being sufficient to suppress clinical symptoms but not sufficient to prevent damage to the infant's myocardium. On the whole the evidence suggested a viral cause for non-familial infant cardiomyopathy. ${ }^{8}$

It could just be possible that these cases represent a heterogeneous group of cardiomyopathies. The mode of death of some infants, the occurrence of similar cardiac conditions in two siblings, and the histological description of vacuolation and perinuclear haloes containing P.A.S.-positive material $^{910}$ are reminiscent of the hypertrophic type of cardiomyopathy. One may speculate (and it has been established in a few instances) that the changes which characterize this condition in older children and adults may take time to develop.

This recent contribution, ${ }^{8}$ like many previous ones, does not show absolute proof of a viral aetiology of cardiomyopathy, but strong suggestive evidence does exist. Viruses can cause myocarditis-for example, Coxsackie virus, ${ }^{11}{ }^{12}$ measles, ${ }^{13}$ mumps, ${ }^{14}$ and influenza. ${ }^{15}$ The transition from incompletely resolved myocarditis-not necessarily viral in origin-to cardiomyopathy has only rarely been substantiated. ${ }^{16}$ Even the presence of raised viral antibody titres is difficult to interpret. G. F. Fletcher and colleagues ${ }^{17}$ investigated serum levels of viral antibody (to Coxsackie virus B1-6, echovirus 6 and 9, influenza $A$ and $B$, mumps, herpes simplex, and psittacosis) in adult patients with primary myocardial disease. These were compared with control subjects. No difference in the titres between test patients and controls was shown. But previous viral infection may be no longer reflected by raised antibody titres and could therefore not be ruled out as the cause of primary myocardial disease. ${ }^{17}$

Thus there is often persuasive evidence that a viral cause seems likely in some cases of cardiomyopathy. The results are frequently difficult to interpret, and further study is necessary to furnish unequivocal proof that viruses are causally related to certain types of cardiomyopathy.

\footnotetext{
${ }^{1}$ Brigden, W., Lancet, 1957, 2, 1179.

Olsen, E. G. J., Cardiovascular Clinics, 4, No. 2, 239, ed. A. N. Brest, and J. E. Edwards, Davis, Philadelphia, 1972

${ }^{3}$ Goodwin, J. F., in Proceedings of $V$ Asian-Pacific Congress of Cardiology, Singapore, in press, 1972.

Oakley, C. M., Postgraduate Medical fournal, 1972, 48, 703

5 Goodwin, J. F., Lancet, 1970, 1, 731.

6 British Medical fournal, 1973, 2, 434.

Gardner, M. B., Lee, P. V., Norris, J. C., Phillips, E., and Caponegro, P., Lancet, 1967, 2, 95 .

${ }^{8}$ Doshi, R., and Lodge, K. V., Archives of Disease in Childhood, 1973, 48 431 .

Van Noorden, S., Olsen, E. G. J., and Pearse, A. G. E., Cardiovascular Research, 1971, 5, 118

${ }^{10}$ Olsen, E. G. J., Postgraduate Medical fournal, 1972, 48, 732.

11 Grist, N. R., and Bell, E. J., British Medical fournal, 1968, 3, 556.
} 